\title{
LOW-VOLTAGE HIGH-SPEED ULTRASONIC CHROMATOGRAPHY FOR MICROFLUIDIC ASSAYS
}

\author{
Chung-Hoon Lee and Amit Lal \\ SonicMEMS Laboratory \\ Department of Electrical and Computer Engineering \\ University of Wisconsin-Madison \\ Madison, WI 53706
}

\begin{abstract}
A novel ultrasonic microfluidic device for polystyrene bead separation is presented. A bead mixture of different size beads are first focused at the pressure nodes in the capillary, and then separated by an inertial nonlinear force generated by capillary transverse vibrations. The separations occur within seconds enabling high speed sample replacement and separations. The bead separation is demonstrated at $1 V_{p p}$ drive at $165 \mathrm{kHz}$ enabling battery powered assays.
\end{abstract}

\section{INTRODUCTION}

A great potential of integrated microfluidic systems is to handle microscale amounts of liquid that bears essential benefits of sample preparation integration, device miniaturization for ambulatory applications, power reduction for battery operation, and lower cost of bioassays. These features can lead to fast diagnostic bio-analytical systems. We present a portable micro analysis system using ultrasonic radiation forces in this paper that promises to fulfill the aforementioned qualities. Cells, proteins, and DNA can all be bound to polystyrene and latex beads. Hence, any system that can separate beads can potentially separate the bio-entities enabling bead based assays. The bead separation technology presented here requires sub five volt operation for CMOS control, and consumes $\sim 10$ milliwatts, and takes less than 10 seconds to separate beads by size. The small platform and low cost of fabrication holds the promise of disposable assay systems.

Figure 1 and Figure 2 describe the acoustic separation system. A glass/polyimide capillary is either wrapped or bonded onto a piezoelectric PZT (lead-zirconate-titanate oxide) plate. The PZT plate stretches the capillary in the length direction which excites the longitudinal mode of the liquid in the capillary leading to bead focusing at pressure nodes in the capillary as discusses later. The PZT can also excite the transverse vibrations of the capillary that separate the focused beads. The focusing and separations occur at locations within the capillary that are highly predictable. Furthermore, the separation occurs over a distance of 100 microns within the capillary enabling easy viewing using a CCD chip as shown in Figure 2. The separation is controlled electronically by tuning the frequency at the PZT making electronic control of the entire assay.

How does the system proposed in this paper compare to other separation methods? Some other techniques for microscale separation in microfluidic systems include electro-osmotic flow, magnetic separations, dielectrophoretic separations, and electrophoretic separations, diffusion-based separations, cantilever-binding mass-sensing assays. A typical electro-osmotic microfluidic assay system for separation utilizing differential mobility in a high electric field for beads/molecules requires a conducting medium and usually high voltages. Magnetic bead separation can require the use of high currents for sufficient magnetic field production or require external magnetics that need to be moved. Dielectrophoretic separations require electrical connections to the fluid and can require non-conducting medium and high voltages. Binding to cantilever and detecting the resulting mass or strain change are also attractive but requires coating of the cantilevers with specific binding molecules.

\section{ACOUSTIC RADIATION FORCE}

Particle manipulation with projected acoustic field has been investigated by many researchers. Acoustic radiation forces generated by concave transducers have been used to concentrate particles at pressure nodes [1]. Previous attempts for focusing beads at pressure nodes using ultrasonic radiation force have led to increased detection limit of agglutination assays [2]. However, to our knowledge, separation of beads by size using the acoustic radiation force has not been demonstrated so far.

The basic principle of the particle manipulation using the acoustic radiation force is that the diffracted acoustic field from a particle leads to a nonsymmetric acoustic field around the particle. The nonsymmetric field results in a net force to move the particle. The $2^{\text {nd }}$ order pressure integrated over the particle surface results in a net force pulling the particles to acoustic nodes or antinodes depending on the acoustic contrast constant,

$$
\mathcal{A}(\alpha, \sigma)=\frac{\alpha+2(\alpha-1) / 3}{1+2 \alpha}-\frac{1}{3 \alpha \sigma^{2}}
$$

where $\alpha$ is the ratio of the bead density to the medium density and $\sigma$ is the ratio of the speed of sound. For standing waves, the radiation force has been derived by Yosioka and Kawasima [3] as,

$$
F_{a c}=-4 \pi k d^{3} \overline{\mathcal{E}} \sin (2 k x) \mathcal{A}(\alpha, \sigma)
$$

where $k$ is the acoustic wave number, $d$ is the radius of a sphere, $\overline{\mathcal{E}}$ is the mean total energy-density, $x$ is the distance from the pressure node and $\mathcal{A}(\alpha, \sigma)$ is the density-compressibility factor that is given by Eq. 1. This expression is valid with $d / \lambda<<1$, where $d$ is the diameter of the bead and $\lambda$ is the wavelength, a condition easily satisfied in experiments. The radiation force $F_{a c}$ has been experimentally evaluated by $\mathrm{K}$. Yasuda and T. Kamakura [4] and found by measuring velocity 
versus time of particles subjected to acoustic pressure. Since the contrast factor $\mathcal{A}$ of polystyrene beads is positive, the density of polystyrene and speed of sound are $1.05 \mathrm{~g} / \mathrm{cm}^{3}$ and 2170 $\mathrm{m} / \mathrm{s}$, respectively, the beads are expected to move toward the pressure nodes $(x=n \lambda / 2$, where $n=0,1,2, \ldots)$ of the standing wave.

In addition to radiation pressure, the volume uptake of the particles in finite space of a glass capillary will lead to the non-linear bands formed by the beads. Since the force is proportional to the cube of the particle diameter $\left(d^{3}\right)$, one predicts that the large particles will move towards the nodes with a higher force, leading to particle separation. Additionally, there is the shear viscous acoustic field near the wall of the glass capillaries that can cause localized streaming and radiation forces but is not important in the effect studied in this paper.

\section{THE GLASS CAPILLARY/PZT ACTUATOR}

A schematic drawing of the glass capillary/PZT transducer is shown in Figure 1. A polyimide coated glass capillary $(\mathrm{ID}=100 \mu \mathrm{m}, \mathrm{OD}=200 \mu \mathrm{m}$, coated polyimide thickness $=12 \mu \mathrm{m})$ is folded around the PZT plate $(10 \mathrm{~mm}$ $\times 2.5 \mathrm{~mm} \times 0.5 \mathrm{~mm}$ ). For intimate contact between the PZT plate and glass capillary, a $300 \mu \mathrm{m}$ deep trench was made on the both ends of the PZT plate and glass capillary is adhesively bonded to the trenches of PZT plate ends using cyanoacrylate. The intimate contact ensures controlled boundary conditions for repeatable results. The bent shape was chosen to ensure easy access to a CCD to image the inside of the capillary. This setup also decouples the acoustic boundary conditions of the separator from those of other components such as the acoustic pump. A typical hand-assembled device is shown in Figure 2. Two electrodes are soldered at the center of the PZT plate since a node of the displacement of PZT plate is at the center.

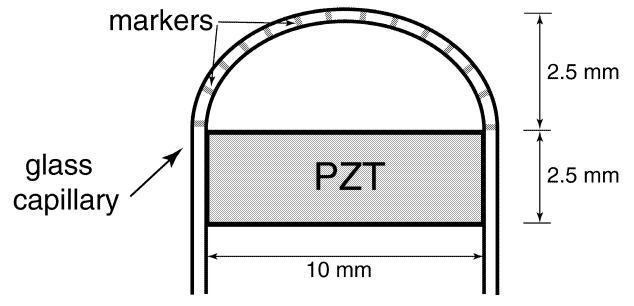

Figure 1. Schematic drawing of the capillary/PZT transducer. The actual device (Figure 2) has a $300 \mu \mathrm{m}$ deep trench at each end of the PZT plate to secure the glass capillary.

The $\lambda / 2$ mode along the PZT plate length is weakly coupled to bending modes as shown in Figure 3 . The resonance frequency of this mode can be approximated as

$$
f=\frac{c}{2 L_{e f f}}
$$

where $c(\sim 3200 \mathrm{~m} / \mathrm{s})$ is the speed of sound in the PZT plate and $L_{e f f}$ is the effective length or width of the PZT plate. From Eq. 3, half-wavelength resonance frequencies for the length mode is $\sim 160 \mathrm{kHz}$. Measured electrical impedance from an HP4194 impedance analyzer is shown in Figure 3.

\section{THE GLASS CAPILLARY MOTION ANALYSIS}

The exact analysis for the capillary motion requires consideration of the finite radius of curvature of the bent

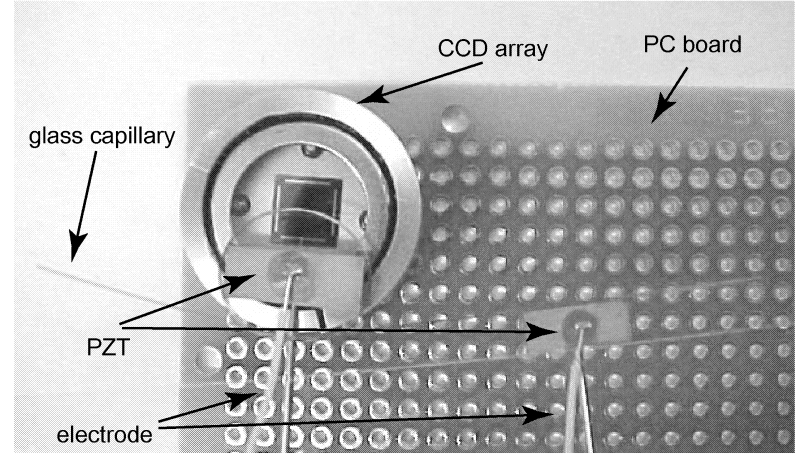

Figure 2. The capillary/PZT transducer with electrodes on a circuit board. There are lithographically defined evenly spaced titanium thin film markers on the capillary to assist velocity measurements and to monitor bead location in the capillary.

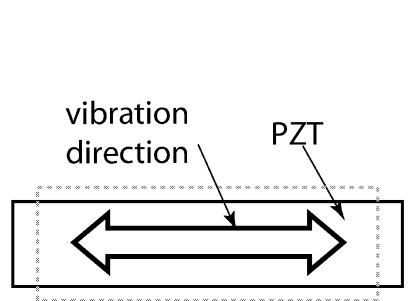

(a) length mode

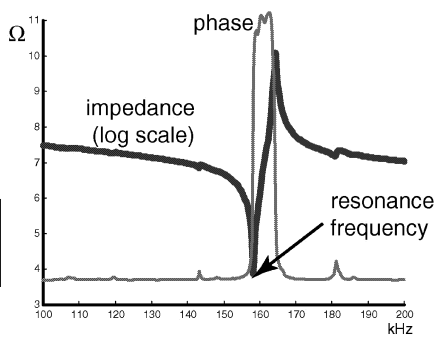

(b) impedance
Figure 3. Measured impedance of the glass capillary/PZT transducer using an HP4194 impedance analyzer (The resonance frequency of the length mode is slightly different from the PZT plate resonance. This is due to the glass capillary loading effect).

beam. Instead we choose to model the bent capillary as a straight beam, which leads to results that reconfirmed by finite element analysis and experimental data. The bending motion of a straight beam can be modelled by

$$
E I \frac{\partial^{4} u}{\partial x^{4}}=\rho A \frac{\partial^{2} u}{\partial t^{2}}
$$

where $E$ is the Young's modulus of the glass capillary, $I$ is the moment of inertia, $\rho$ is the density, $A$ is the cross section area, and $u$ is the displacement. The standing wave solutions to Eq. 4 can be written as

$$
\begin{aligned}
u(x)= & C_{1} \sinh (k x)+C_{2} \cosh (k x) \\
& +C_{3} \sin (k x)+C_{4} \cos (k x)
\end{aligned}
$$

For the clamped-clamped beam, the $\sin (k x)$ dominates while the $\sinh (k x)$ satisfies boundary conditions at the clamped edges. Here we approximate the solution as $\sin (k x)$ for simplicity. In addition to capillary bending modes, the motion of the PZT plate is superposed on the capillary mode. As shown in Figure 4, if the capillary is not resonated, the PZT plate motion induces the following approximate motion to the capillary,

$$
\begin{aligned}
v_{x} & \sim x / L-1 / 2 \\
v_{y, d c} & \sim-x \cdot(x-L)
\end{aligned}
$$


where $L$ is the length of the capillary and PZT plate edge is located at $x=0$. However, if the capillary is resonated at its bending mode resonance frequencies, an AC motion of the glass capillary will be induced as mentioned above (Eq. 5) and can be written as

$$
v_{y, a c} \sim \sin (k x)
$$

Hence, the total glass capillary velocity in the $y$ direction may be written as,

$$
v_{y, \text { total }}=A_{1} \cdot x \cdot(x-L)+B_{1} \cdot \sin (k x)
$$

where $A_{1}$ and $B_{1}$ are constants determined by device dimensions, material properties and driving frequency.

The total velocity profile of the capillary in the $x$ and $y$ directions are shown in Figure 4 for $A=6$ and $B=1$.

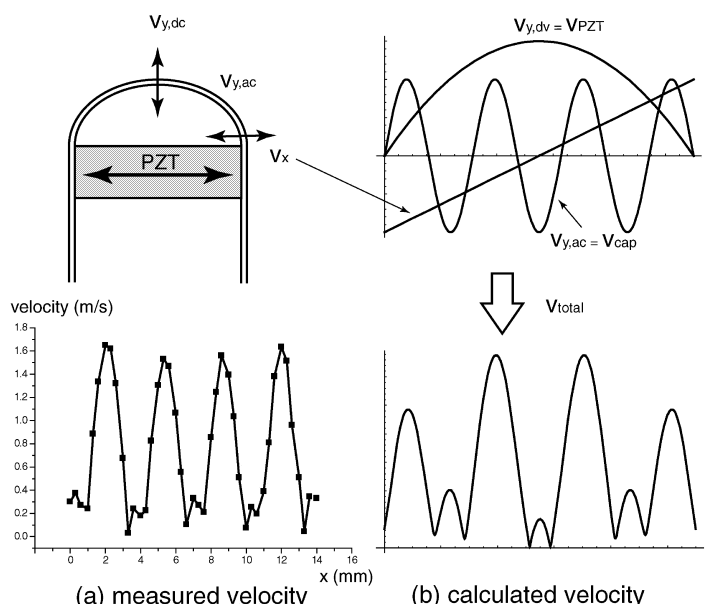

Figure 4. Measured $v_{\text {total }}$ and analytical profile of $v_{x}, v_{y}$ and $v_{\text {total }}$ using straight-beam approximation.

The capillary displacement and velocity were measured using a Polytec-PI interferometer. The measured velocity of the capillary excited at $160 \mathrm{kHz}$ (corresponds to PZT $\lambda / 2$ mode) are shown in Figure 4 (a). This measured velocity profile matches well with the calculated data. ANSYS analysis was also done to calculate the capillary transverse motion, for comparison with the measured data as shown in Figure 5. The calculated velocity is well matched with the measured data and the simple model except near the capillary-PZT plate joint. It is believed that this discrepancy was due to pre-existing tension of the bent capillary that was not included in the analysis. Modal analysis revealed that there are several resonance modes which correspond to capillary resonance and total system resonance (Figure 6). As we can clearly see in Figure 6 the capillary resonance (for example, $\sim 139$ and $166 \mathrm{kHz}$ ) is close to the total system resonance $(\sim 159 \mathrm{kHz})$ as also verified by experiments.

\section{PRINCIPLE OF OPERATION}

Given the motion analysis, we can focus on how the acoustic system separates particles. The transverse motion of the capillary is excited over a wide range of frequencies as shown in Figure 6. This motion induces a force given by

$$
F_{\text {inertial }}=\frac{\pi^{2}}{4} M u_{0}^{2} \omega^{2} k_{c a p} \sin \left(2 k_{c a p} x\right)
$$
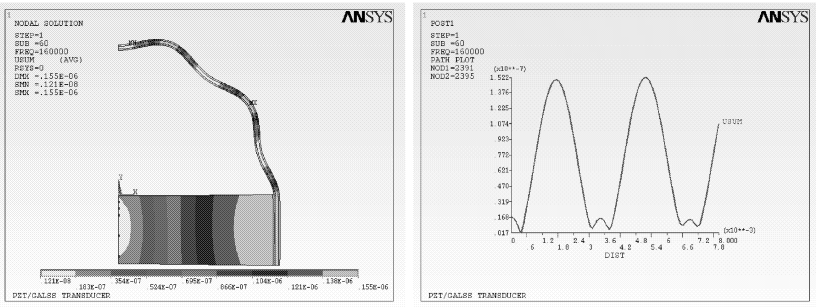

Figure 5. ANSYS harmonic analysis. (a) The motion of the actuator. (b) The displacement profile along the capillary.

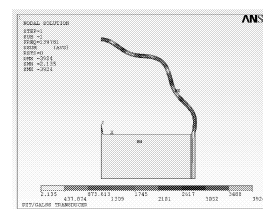

(a) $139 \mathrm{kHz}$

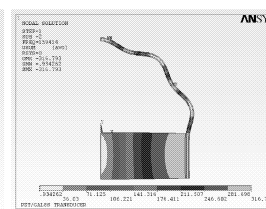

(b) $159 \mathrm{kHz}$

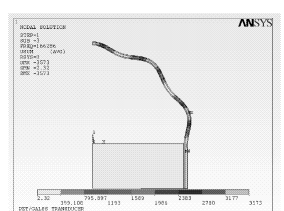

(c) $166 \mathrm{kHz}$
Figure 6. ANSYS modal analysis results. (b) indicates glass capillary/PZT coupled resonance. Other modes are PZT motion exciting eigen modes of capillary.

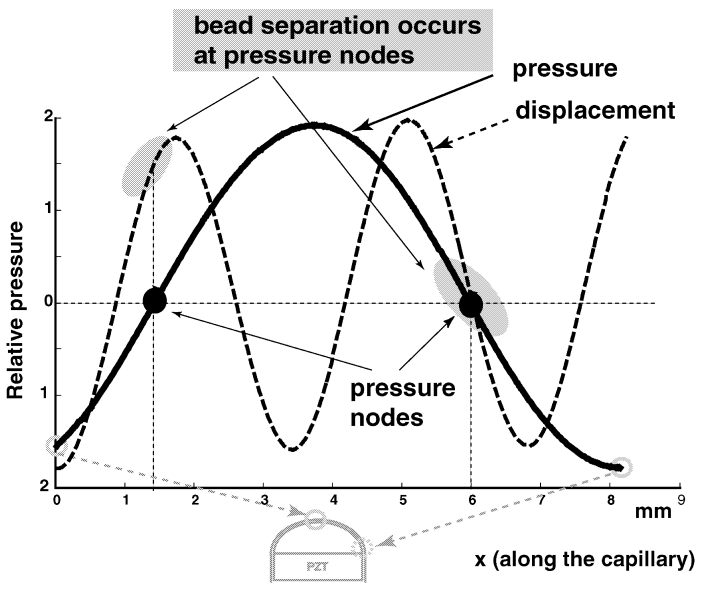

Figure 7. ANSYS derived the pressure profile inside the capillary: The displacement profile of the capillary was overlapped onto the pressure profile. the polystyrene beads were focused at the pressure nodes and separated.

where $\mathrm{M}\left(=\frac{4 \pi}{3} d^{3}\left(\rho_{\text {bead }}-\rho_{\text {water }}\right)\right)$ is the mass difference between the bead and water, $u_{0}$ is the transverse displacement amplitude, $\omega$ is the radian frequency, and $k_{c a p}$ is the wavenumber corresponding to the capillary mode. The derivation of this force is beyond the scope of this paper. The inertial force alone randomizes the bead motion and separation is not achieved. To separate, the radiation force along the capillary is needed. The x-directed velocity in Figure 4 is a approximated as a straight line. The PZT stretches the capillary exciting the symmetric acoustic pressure standing waves inside the capillary. This pressure wave is shown as the pseudo-half wavelength pressure shown in Figure 7. This pressure wave is responsible for focusing the beads at the pressure nodes as predicted by Eq. 2. At PZT resonance, this force is sufficient to overcome the inertial forces described by Eq. 10. Then as the frequency is changed slightly around the resonance frequency, the radiation force is reduced and the inertial force acting 
in different directions separates the beads at different angles as shown in Figure 8. Hence,bead separation is enabled by application of radiation and inertial forces acting in different directions with controlled amplitudes. As shown in Figure 7, the inertial force is maximized at the points of maximum slope of the capillary transverse motion, close to the points of stable points of radiation force. Two such points are shown in Figure 7, which were verified by experiments.

\section{SEPARATION EXPERIMENTS}

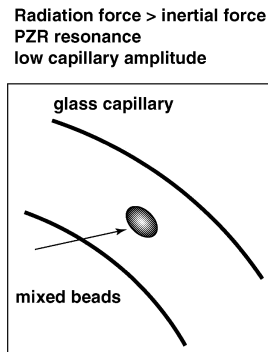

(a)

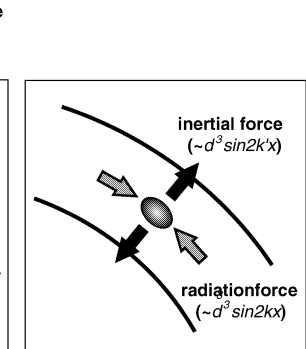

(b)

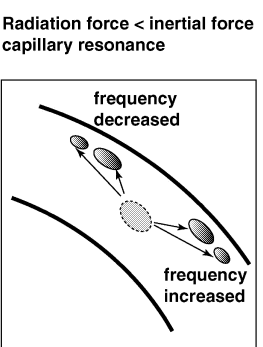

(c)
Figure 8. (a) The radiation force acting axially focuses all the beads together overcoming the inertial forces. (b) The radiation force reduction and the separation of particles. Note that as the frequency is increased or decreased, the inertial force vector changes giving separated beads in two different angles as shown in (c).

Although different bead sizes ranging from $0.5 \mu \mathrm{m}$ to $10 \mu \mathrm{m}$ were separated, the methodology is displayed for 3 and $10 \mu \mathrm{m}$ beads is shown in Figure 9. Figure 9 (a) shows uniformly distributed bead mixture in the capillary before the actuation, (b) shows the beads moving to a node when the actuator is driven at the PZT plate length mode $(159 \mathrm{kHz} @$ $1 V_{p p}$ ) resonance resulting focusing of the beads at the pressure nodes. After the beads moved to the node, the frequency is changed to $165 \mathrm{kHz}$ resulting in bead separation due to the intertial force acting in orthogonal directions to the radiation force (c), and then 3 seconds later the bead mixture was totally separated, (d). Such beads separations could be used to perform ratiometric assays if beads are coated by different antibodies. Another application of separation is to separate white cells from red blood cells for diagnosis. Figure 10 shows the left over white blood cells after red blood cells were lysed away in the bead focus areas.

\section{CONCLUSIONS}

A novel structure for bead separation for assays using radiation pressure and inertial forces has been demonstrated. An analysis of capillary motion that agrees with the measured data leads to pressure nodes where the beads move into and the radiation force and the gradient of the radiation force separate different size beads. The driving voltage was less than $1 V_{p p}$, which can be operated by traditional CMOS circuits. The glass capillary/PZT actuator was successfully integrated onto a $\mathrm{CCD} / \mathrm{LCD}$ (with a portable computer) for a portable microfluidic assay system.

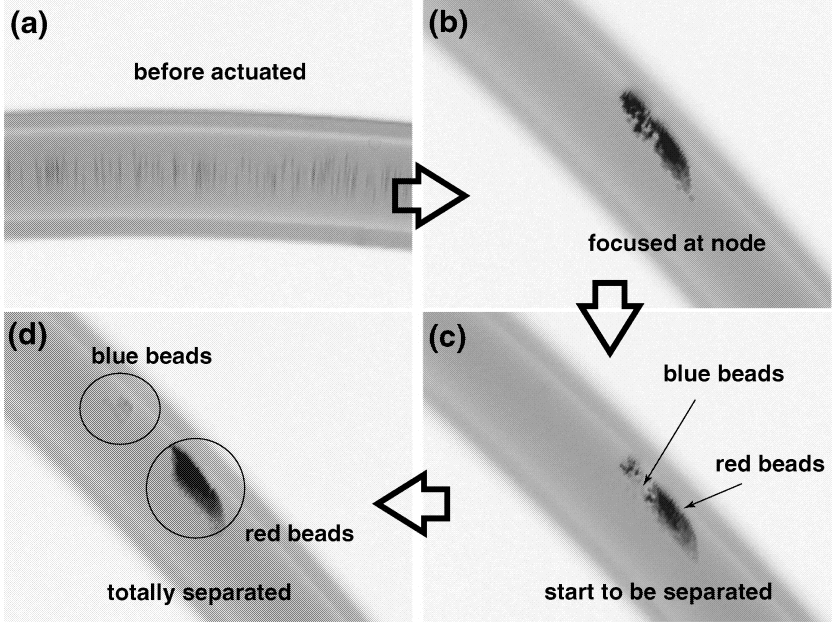

Figure 9. Bead separation at a pressure node: the size of the beads on top-left side is $3 \mu \mathrm{m}$ and on bottom-right side is $10 \mu \mathrm{m}$ in (d). These pictures are taken on a fluorescence microscope to distinguish the beads by color.

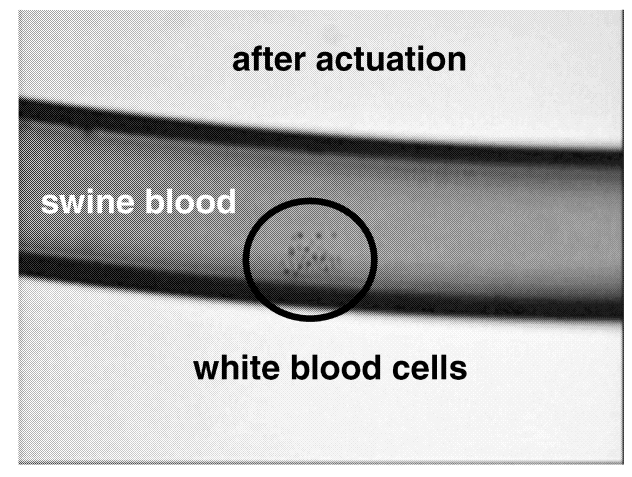

Figure 10. A portable glass capillary/PZT actuator assay system: Swine white cell separation.

\section{ACKNOWLEDGEMENTS}

We would like to thank Wisconsin Center for Advanced Microelectronics (WCAM) for technical support. This reserach was supported in part by DARPA-MTO under the BioFlip program, contract \#F30602-00-2-0572.

\section{REFERENCES}

1. T. Kozuka et al., "Non-contact micromanipulation using an ultrasonic standing wave field," IEEE, The Ninth Annual International Workshop on Micro Electro Mechanical Systems, pp. 435-440, 1996.

2. G. Whitworth and W. T. Coakley, "Particle column formation in a stationary ultrasonic field," Journal-of-theAcoustical-Society-of-America, vol. 91, no. 1, pp. 79-85, 1992.

3. K. Yosioka and Y. Kawasima, "Acoustic radiaotion pressure on a compressible sphere," Acustica, vol. 5, pp. 167-173, 1955.

4. K. Yasuda and T. Kamakura, "Acoustic radiation force on micrometer-size particles," Appl. Phys. Lett., vol. 71, no. 13, pp. 1771-1773, 1997. 